\title{
The Algebraical Superposition Technic for Transformation from S Domain to Time Domain
}

\author{
Benqing Gao \\ Beijing Institute of Technology, School of Information Science and Technology, Beijing, China
}

\section{Email address:}

gaobq@bit.edu.cn

\section{To cite this article:}

Benqing Gao. The Algebraical Superposition Technic for Transformation from S Domain to Time Domain. American Journal of Modern Physics. Vol. 9, No. 4, 2020, pp. 55-59. doi: 10.11648/j.ajmp.20200904.11

Received: June 9, 2020; Accepted: July 7, 2020; Published: August 17, 2020

\begin{abstract}
An algebraical superposition technic for transformation from frequency domain to time domain is presented. The establishing model process are; From the inverse Laplace transform integral formula, the integral formula can be expressed by the series with $\mathrm{k}[-\mathrm{K}, \mathrm{K}]$ term., based on integral function distribution properties along integral path, the bidirectional series sum on $\mathrm{k}[-\mathrm{K}, \mathrm{K}]$ term series can be expressed by a monomial trigonomial function series sum on $\mathrm{k}[0, \mathrm{~K}]$. In the paper the solution process and main points are presented. The application examples are shown; the results are supported to the algebraical superposition technic. In using above algebraical superposition technic to analysis airspace dispersive propagation properties, i.e., to find out its time-domain parameters, the resultant formula contain time-domain factor and frequency-domain factor, that is first time to present in the paper, so it is called, 'time-frequency union technic'. In the paper simultaneity solve out wave number and wave impendance for waveguide TE10 mode propagation, and get both time-frequency union values, and show the soving process and soving accuracy.
\end{abstract}

Keywords: Frequency Domain to Time Domain Trasformation, Laplace Transformation, Algebraical Superposition Technic, Rectangular Wavwguide TE10 Mode, Time-frequency Union Value

\section{Introduction}

In scitific and technic zone for electronics and dynams and controls, etc. It is often necessary to observe and research a system parameters varying to time. In mean time, it is often necessary to know a system paramters varying to frequency. The former is called as time domain paramters, and the later is called frequency domain paramters. It can be knowed from one to another by the trasformation between times domain to frequency domaim. The used time domain to frequency domain trasformation method is often the Fourier Transform (FT) and Laplace Transform (LT). The condition using LT is loose, so its use is wider. The knowledge on Laplace Transform is easily found [1-3].

Implementating Laplace Transform from frequency domain to time domain an algebraical superposition technic is presented in the paper. It is from the inverse Laplace transform integral formula, the integral formula can be expressed by the series with $\mathrm{k}[-\mathrm{K}, \mathrm{K}]$ term. When the amplitude and phase values for the series are even and odd symmatry to $\mathrm{k}=0$ respectively, the bidirectional series sum on $\mathrm{k}[-\mathrm{K}, \mathrm{K}]$ term series can be expressed by a monomial trigonomial function series sum on $\mathrm{k}[0, \mathrm{~K}]$. In the paper the solution process and main points are presented. The application examples are expressed; the resules are supported to the algebraical superposition technic.

Foremore, we research on the transformation of transmission parameters (frequency domain). In dispersive space domain has done. It is: in TE10 mode waveguide [4-6] the transformation of transmission parameters. Its main process is: starting from the itegral formula of inverse Laplace transform, it can be expressed by it's a set of equivalent series with $\mathrm{k}[1, \mathrm{~K}]$ term.... After driving we find the last output sequence is consisted of two elements: the one is time factor, the two is frequency factor. In the inverse Laplace transform the results are not appeared. So it is called: the new idea and method of 'time-frequency union value'. The propagation wave number and the wave impedance of TE10 mode waveguide are calculated in paper. The results are suportted to above research.

In the paper, all calculation and program and graph are all performed by author himself 


\section{General Problem}

\subsection{Calculation Mode}

Now, from Laplace transform formula research the calculation process of setting up, as follows

The first, from inverse Laplace transform formula [7-9].

$$
f(t)=\frac{1}{2 \pi j} \int_{\sigma-j \infty}^{\sigma+j \infty} F(s) e^{s t} d s \quad s=\sigma+j \omega \quad \omega=2 \pi f(1)
$$

The coresponding Laplace transform formula is

$$
F(s)=\int_{-\infty}^{\infty} f(t) e^{-s t} d t
$$

Above the itegral for function $f(t)$ can be expressedBy it $\mathrm{s}$ series sum in the following:

$$
f\left(t_{i}\right)=\Delta f \sum_{k=-K}^{K} F(s) e^{s t_{i}} \quad k=-K, \ldots,-2,-1,0,1,2, \ldots, K
$$

Foremore, $F(s)$ in (3) ofen be complex and be expressed by its amplitude and phase $\left(F_{m}(k) e^{j \phi_{k}}\right)$, its $e^{s t i}$ can also be expressed by its real and imaginary part $\left(e^{\sigma t_{i}} e^{j \omega_{k} t_{i}}\right)$, and $k[-K, K]$ is divided as three parts: $[-K,-1], 0,[1, K]$, as follows:

$$
\begin{aligned}
& f\left(t_{i}\right)=\Delta f\left\{F_{m}(0) e^{j \phi_{0}} e^{\sigma t_{i}}+\left[\sum_{k=-K}^{-1}+\sum_{k=1}^{K}\right] F_{m}(k) e^{\sigma t_{i}} e^{j \omega_{k} t_{i}} e^{j \phi_{k}}\right\} \\
& =\Delta f e^{\sigma t_{i}}\left\{F_{m}(0) e^{j \phi_{0}}+\left[\sum_{k=-K}^{-1}+\sum_{k=1}^{K}\right] F_{m}(k) e^{j\left(\omega_{k} t_{i}+\phi_{k}\right)}\right\}
\end{aligned}
$$

At last, when frequency domain function $F(s)$ distribution chacteristics are: $F_{m}(k)=F_{m}(-k), \phi_{m}(k)=-\phi_{m}(-k)$, By Urla equation $e^{j \varphi}+e^{-j \varphi}=2 \cos \varphi$ we obtain

$$
\begin{gathered}
f\left(t_{i}\right)=\Delta f e^{\sigma t_{i}}\left\{F_{m}(0) e^{j \phi_{0}}+2 \sum_{k=1}^{K} F_{m}(k) \cos \left(\omega_{k} t_{i}+\phi_{k}\right)\right\} \\
i=0,1,2, \ldots \ldots, N_{t}
\end{gathered}
$$

Above (5) is our request formula for calculation.

\subsection{Kernel of Implematation}

From equation (5) respective time domain sequence can be found, the kenel of implematation are:

(a) The analysis of respective frequency domain function: when the region of frequency domain and sampling space $(\Delta f)$ are given, $F_{m}(k), F_{m}(-k)$ and $\phi_{m}(k), \phi_{m}(-k)$ are calculated which are used to judge $\left(F_{m}(k)=F_{m}(-k)\right.$, $\left.\phi_{m}(k)=-\phi_{m}(-k)\right)$, if it is satisfied, the equation (5) can be used to find out the result.

(b) The time domain sequences sampling spacing and calculation region for frequency and time domain are determined:

By $\Delta f, N_{f}$ are respectively expressed for sampling spacing and sampling poimt number in frequency domain, $f_{h}$ and $f_{s}$ being the highest and sampling frequency respectively, Its well known that we have:

$$
\Delta f=\frac{1}{t_{s}}=\frac{f_{s}}{N_{f}} ; \quad f_{s}=(3-6) f_{h}=\frac{1}{\Delta t} ; \quad ; \Delta t \leq \frac{1}{2 f_{h}}(6)
$$

(c) Main point for time domain calculation: when calculation by equation (5), the intergal path is a line that is parallel to imaginary axis and its real value being $\sigma$. The half axis line is equially divided, so obtained length $\Delta f$ with $K$. Theoretically, $K$ value approximate to $\infty$, but in practice calculation it can be determinated by the following way. The first $K_{i}$ is given and a set of tine domain data is obtained from equation (5). Next, $K_{i+1}$ is given and another of tine domain data is obtained. Comparising the two set of tine domain data, if there are two digit values or more two digit values being equal, we can approximately think that $K_{i+1}$ set of time domain data is 'convegence value' of finding time sequence.

(d) To get the 'convegence value' of the time sequence. It is nessercery to calculate the 'convegence value'with algebraically superposit for hundreds or thousands or ten thousands or more times. To keep the accuracy of calculation data, we use the double accuracy for datas

(e) It is necessary to verify the time domain solution (time domain sequence). The verification method is: the solution sequence though transformation to get its frequency domain values (called calculation value). The frequency domain sequence from frequency domain function $F(s)$ is called as theory value. Comparing the calculation value to the theory value, the conclosion will come.

\subsection{Aplication Examples}

Two examples will be adopted; implementing the transform from frequency domain to time domain [7-12] above Kernel of implematation will be followed. On its individuality the simple word will express.

*** Example 1.

$$
F(s)=\frac{\sqrt{s^{2}+6 s+7}}{(s+1)(s+4)}, \quad s=\sigma+j \omega, \quad \omega=2 \pi f
$$

The solution of the example can not be obtained by general method, but can be obtained by equation (5). The main process are: $\sigma$ and parameter for calculation using in frequency domain and time domain are determinated, they are: $\sigma=-2.5 ; \Delta f=500 \mathrm{~Hz}, f_{h}=3 \times 10^{4} \mathrm{H}_{z} ; \Delta t=1 \times 10^{-5} \mathrm{~s}$, $N t=200$.

The first, $\quad F_{m}(k), F_{m}(-k)$ and $\phi_{m}(k), \phi_{m}(-k)$ are numerated, and their distribution case are $F_{m}(k)=F_{m}(-k)$, $\phi_{m}(k)=-\phi_{m}(-k)$. which will make us to obtain $\mathrm{f}(\mathrm{t})$ from 
formula (5). And the frequency domain sequence is theory value of this example.

Next, the expectant time domain sequence can be got by equation (5). To obtain time domain sequence convegence value, in calculation, incresing $\mathrm{K}$ step by step, $K=5000$ can be taken as convegence value. As comprasing time domain sequence for $K=5000$ to $K=4000$, the two sequence values that have same value in 2-3 digits. It is shown in Figure 1 (a)

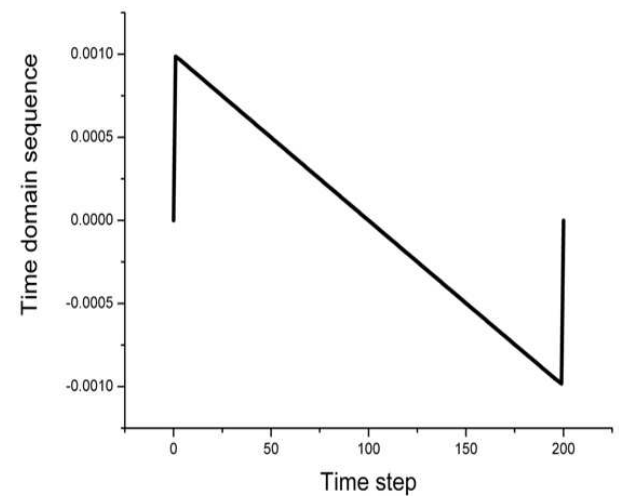

(a)

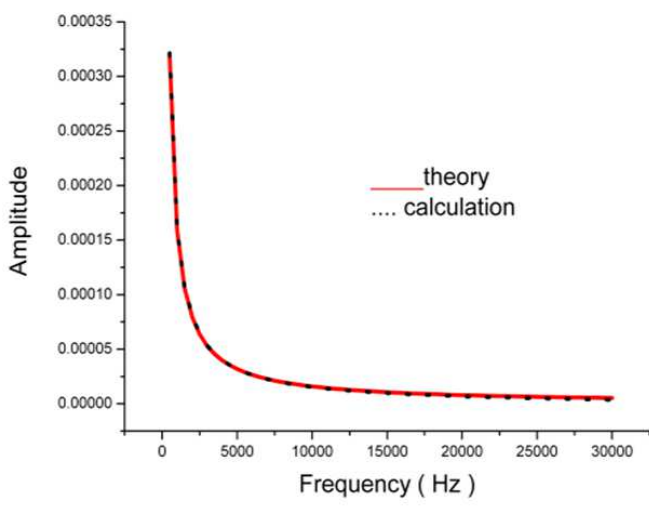

(b)

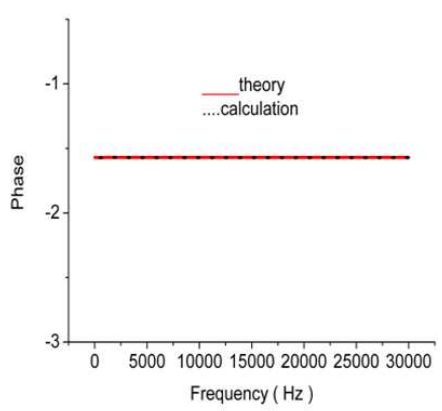

(c)

Figure 1. Variation curve figure for example 1.(a) Time domain sequence; (b) ampletude; (c) phase.

****xample 2.

$$
F(s)=\frac{1}{\sqrt{s^{5}+4 s^{4}+8 s^{3}}+8 s^{2}+7 s+4}, \quad s=\sigma+j \omega, \quad \omega=2 \pi f(8)
$$

This is a mixed fraction of rational and irrational polynomial; it can not be obtained by general method. It can be solved by formula (5). The parameters are chose: $\sigma=-2.0$; $\Delta f=0.1 \mathrm{~Hz}, f_{h}=6 H_{z} ; \Delta t=5 \times 10^{-2} s, N t=200$.[1] [2] [3]. After calculation for $F(s)$ it have $F_{m}(k)=F_{m}(-k)$, $\phi_{m}(k)=-\phi_{m}(-k)$ this frequency domain sequence $(k=0,1$, $2, N_{t}$ ) is just the theory value of the formula (8)

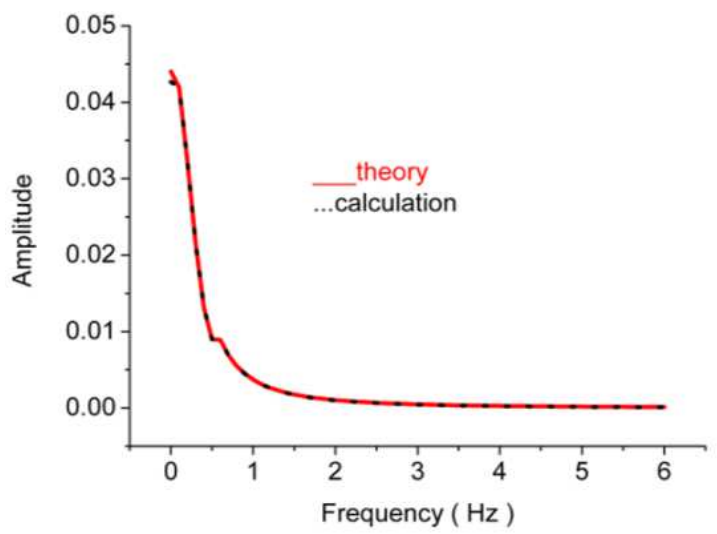

(a)
Implimantating time domain solution calculation, the time domain sequence for $K=2000$ can be taken

As the convegence values, for the values have two or more digits with $K=1500$. The spectrum of the time domain sequence is calculation value.

Comparison calculation value with theory value $(F(s))$ are shown in Figure 2 (a) and (b). The amplitude and phase are shown respectively in (a) (b) figures. The two are well agreement in $f=0.1 \rightarrow 6.0 \mathrm{~Hz}$. The phase value variation with frequecy are much large (in (b)) i.e., they are went down abruptly at $f=1.4 \mathrm{~Hz}, 1.5 \mathrm{~Hz}$ (from 3.1 to $-3,1$ )

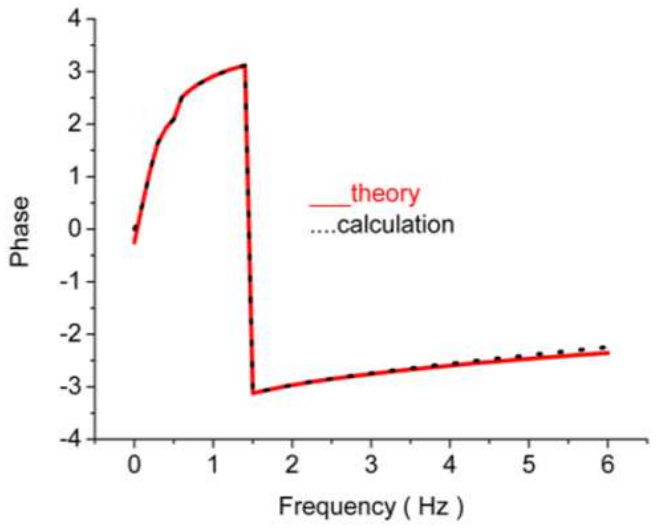

(b)

Figure 2. Comparison betreen theory and calculation.(a) Amplitude;(b) phase.

\section{Dispersive Space}

\subsection{Mode}

Research how to establish time domain sequence calculation mode from the example of limit frequency band. The example is a rectangular waveguide with disperce property. We know [4]: waveguide sizes are $\mathrm{a}=0.02286 \mathrm{~m}$, $\mathrm{b}=0.01016 \mathrm{~m}$, which working in $\mathrm{x}$ band for TE10 (H10) mode. Its effect working frequency is $\mathrm{f}=0.6633 \sim 1.3204 \times 10^{10} H_{z}$. 
Its main frequency domain parameter is the propagation wave number $\Gamma_{10}$ for TE10 (H10) mode

$$
\begin{gathered}
\Gamma_{10}=j \beta_{10}, \quad \beta_{10}^{2}=k_{0}^{2}-k_{c, 10}^{2}=\frac{\omega^{2}}{c^{2}}-\frac{\pi^{2}}{a^{2}} \\
\beta_{10}(s)=F(s)=\sqrt{\frac{\omega^{2}}{c^{2}}-\frac{\pi^{2}}{a^{2}}}, s=j \omega \omega=2 \pi f
\end{gathered}
$$

From above frequency domain parameter to find out its time domain sequence, the inverse Laplace transform formula is used as follows

$$
\begin{aligned}
\beta_{10}(t) & =\frac{1}{2 \pi j} \int_{0}^{j \infty} \beta_{10}(s) e^{s t} d s=\frac{1}{2 \pi j} \int_{0}^{j \infty} \sqrt{\frac{\omega^{2}}{c^{2}}-\frac{\pi^{2}}{a^{2}}} e^{j \omega t} j 2 \pi d f \\
& =\int_{0}^{j \infty} \sqrt{\frac{\omega^{2}}{c^{2}}-\frac{\pi^{2}}{a^{2}}} e^{j \omega t} d f
\end{aligned}
$$

In above equation $s=j \omega, \sigma=0$. as it is often the air in waveguide region. The integral formula $\beta_{10}(t)$ can be expressed by series sum formula, and its working frequency region is considered, we obtain:

$$
\beta_{10}(i)=\sum_{k=1}^{K} \sqrt{\frac{\omega_{k}^{2}}{c^{2}}-\frac{\pi^{2}}{a^{2}}} \exp \left(j \omega_{k} t_{i}\right) \Delta f \quad i=0,1,2, \ldots . N_{t}(10)
$$

Just is time domain sequence iterm.

When $\Delta f=0.7143 \times 10^{8} \mathrm{~Hz}$, the point number of working frequency domain sequence is $\mathrm{k}=92$. In above equation the main factor is: $\sqrt{\frac{\omega_{k}{ }^{2}}{c^{2}}-\frac{\pi^{2}}{a^{2}}}$ and $\exp \left(j \omega_{k} t\right)$. The second is the function of $\omega_{k} t$, but the first is only the function of $\omega_{k}$, it can be expressed by $\beta_{10}\left(f_{k}\right)$

$$
\beta_{10}\left(f_{k}\right)=\sum_{1}^{k} \sqrt{\frac{\omega_{k}^{2}}{c^{2}}-\frac{\pi^{2}}{a^{2}}} \Delta f
$$

So the formula (10) can be expressed as follows

$$
\beta_{10}(i)=\beta_{10}\left(f_{k}\right) \exp \left(j \omega_{k} t_{i}\right) \quad i=0,1,2, \ldots N_{t}
$$

The formula (12) is just last formula for calculation.

\subsection{Main point for Calculation}

Analysis of formula (11) (12):

(a) Time domain sequence (12) contains two iterms: the first is formula (11), The second item $\exp \left(j \omega_{k} t\right)$ that is just time sequence iterm.

(b) Time sequence iterm can be calculated from the second iterm of formula (12). Analysis and calculation show: the spectrum sequence peak value is located at $\omega_{k}\left(f_{k}\right)$, At other frequency point that is away from $f_{k}$, the spectrum sequence values are approximately a contant.

(c) As saying in (a), the spectrum calculation value for TE10 mode of the rectangular waveguide is equal to the mutiply of the second iterm (the spectrum of time domain sequence) with the first iterm (TE10 mode wave number value in frequency domain). Therefore, the spectrum sequence calculation value can be thinked as departure cooeficint between calculetion and theory value. Practical calculation show: these cooeficient values are approximate 1 at several $\Delta f$ frequency points apart from $f_{k}$.

(d) In analysis the frequency domain factor and time domain factor are expressed at one time in equation (12). This result is not appearing in generl materials. Therefore, this analysis teachnic is called as new idea and new method of 'time-frequency union value'. The calculation results (expressed in 3.3) show: it is rational.

\subsection{Calculation Results}

This time the centre frequency: $\mathrm{f}=9.84778 \times 10^{10} \mathrm{H}_{z}$, Parameters for calculation:

$$
\Delta t=0.14 \times 10^{-10}, \quad N_{t}=1000 ; \quad \Delta f=0.7143 \times 10^{8}, N_{f}=92
$$

The calculation result is: at centre frequency the spectrum sequence peak value is 1001 . At each frequency points that depart fromthe centre point is over 5 frequency points, the spectrum sequence peak value is 1 approximately.

To avoid peak value 1001 appearing at work band $k$ should be taken that is higher than up-limit work frequency, i.e., $f_{k}=f_{107}=1.4205 e 10 \mathrm{~Hz}$. The calculation results are shown in Figure 3. From it, we can see: at effect work frequency $\mathrm{f}=$ $0.6633 \sim 1.3204 \times 10^{10} H_{z}$ its spectrum variation case. i.e., the mode value of complex sequence variation are betreen $1.019 \sim$ 1.022. It is the error between theory and calculation is less than $2.5 \%$.

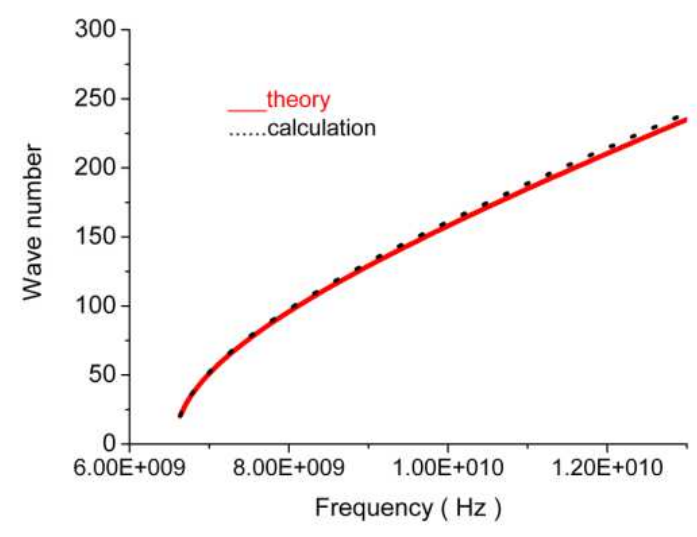

Figure 3. Comparison between theory andcalculation of the wave numbere of waveguide TE10 mode. 


\subsection{Wave Impedance Analysis and Calculation for Rectangular Waveguide TE10 Mode}

Wave impedane equation for rectangular waveguide TE10 mode as follows [3]:

$$
Z h_{10}(f)=\frac{k_{0}}{\beta_{10}(f)} z_{0}=\frac{k_{0}}{\beta_{10}(f)} \sqrt{\frac{\mu_{0}}{\varepsilon_{0}}}=\frac{\omega \mu_{0}}{\beta_{10}(f)}(13)
$$

Above equation $\beta_{10}(f)$ is wave propagation number. After the same process to above process, the equation for the time domain sequence of wave impedance can be obtained in the following

$$
\begin{aligned}
Z h_{10}(i)=Z h_{10}\left(f_{k}\right) \exp \left(j \omega_{k} t_{i}\right) \quad & i=0,1,2, \ldots . N_{t} \\
Z h_{10}\left(f_{k}\right) & =\sum_{k=1}^{k} \frac{\omega \mu_{0}}{\beta_{10}} \Delta f
\end{aligned}
$$

The equation (15) is only the frequency domain sequence

The same process to the propagation wave number, this soving process is also called: new method of 'time-frequency joint value'.

In process for finding out time domain sequence of wave impedance, the calculation paramters are same as above. The calculation result is expressed in Figure 4. It can be seen: in $\mathrm{f}=$ $0.6633 \sim 1.3204 \times 10^{10} H_{z}$. The complex number sequence mode value variation region is $1.019 \sim 1.022$, i.e., the error betrewn calculation and theory mode value is less than $2.5 \%$.

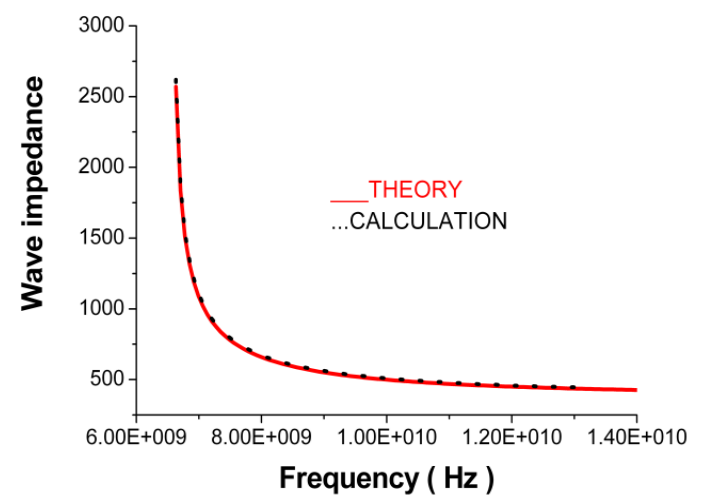

Figure 4. Comparison between theory and calculation value of the wave impedanceof waveguide TE10 mode.

\section{Conclution}

(1) The algebraical superposition technic presented is a simple and easily implementation. It is applicable for rational and irrational function transformation.

(2) When the algebraical superposition technic is used in the of dispersive space problem, i.e., the analysis for propagation parameters of rectanglar waveguide, The time domain solution obtained is the product of the time domain factor and the frequency factor. The extraordinary solution is first appear in the paper, is called 'Time-frequency Union Value'.

(3) 'time-frequency union technic' is a bright point in the paper

\section{Acknowledgements}

This work was carried out at the EM Simulation Lab I am grateful the constant assistance and encouragement of Professer Li wen ming, Ren wu, Xun zheng hui, without whom this work could not have been completed.

\section{References}

[1] Zheng Jun li, Signal and system introduction, Beijing, High education publisher, 2009, p 420-438

[2] Wu xiang qi, Signal and system (third), Beijing, Electronic industry publisher, 2009, p 338.

[3] Zeng Yu Cun deng, Signal and system (third), Beijing, Beijing Institute of Technology publisher, 2010, p. 266-283.

[4] (USA) R. E. Collin, Translation: Chinese, Hou Yuanqing, Field theory of guided waves, Shanghai Science-technic Publisher, 1960 , p 182-184.

[5] Lu Shanwei, Microwave Engineering Foundation, Beijing aviation and spaceflight university publisher, 1995, P. 128-139.

[6] Yan Yun Qing, Li Ying Hui, Micriwave Technic Fondamention (fourth), Beijing Instituteof Technology Publisher, 2011, P. 68-96.

[7] Liang Kun Miao, Mathematic Physic Method, Beijing Education Publisher, 1978, p. 85-99.

[8] Fu Dan University Mathematic Depatment Editor, Mathematic PhysicEquation (second), ShanghaiScience Technic Publisher, 1961, p. 78-94.

[9] Mathematic handbook writing group, Mathematic handbook, Beijing, Pepole education publish, 1979, p. 553-564.

[10] Po Go Neng, Fortran 95 Program Design, Beijinb, China electric power publisher, 2006, p. 562-567.

[11] Ben-Qing Gao, An algebraical superposition technic for transformation from $\mathrm{Z}$ domain to time domain, 2018, WJRR, Vol. 6, May, P. 4-7.

[12] Ben-Qing Gao, A new priciple technic for the transformation from frequency to time domain, 2017, AIP Advances 7, 035315, USA.. 